\title{
Editorial
}

\section{Nonlinear Analysis of Dynamical Complex Networks}

\author{
Zidong Wang, ${ }^{1,2}$ Bo Shen, ${ }^{1}$ Hongli Dong, ${ }^{3,4}$ and Jun $\mathrm{Hu}^{4,5}$ \\ ${ }^{1}$ School of Information Science and Technology, Donghua University, Shanghai 200051, China \\ ${ }^{2}$ Department of Information Systems and Computing, Brunel University, Uxbridge, Middlesex UB8 3PH, UK \\ ${ }^{3}$ College of Electrical and Information Engineering, Northeast Petroleum University, Daqing 163318, China \\ ${ }^{4}$ Research Institute of Intelligent Control and Systems, Harbin Institute of Technology, Harbin 150001, China \\ ${ }^{5}$ Department of Applied Mathematics, Harbin University of Science and Technology, Harbin 150080, China
}

Correspondence should be addressed to Zidong Wang; zidong.wang@brunel.ac.uk

Received 10 October 2013; Accepted 10 October 2013

Copyright (C) 2013 Zidong Wang et al. This is an open access article distributed under the Creative Commons Attribution License, which permits unrestricted use, distribution, and reproduction in any medium, provided the original work is properly cited.

Complex networks are composed of a large number of highly interconnected dynamical units and therefore exhibit very complicated dynamics. Examples of such complex networks include the Internet, that is, a network of routers or domains, the World Wide Web (WWW), that is, a network of websites, the brain, that is, a network of neurons, and an organization, that is, a network of people. Since the introduction of the small-world network principle, a great deal of research has been focused on the dependence of the asymptotic behavior of interconnected oscillatory agents on the structural properties of complex networks. It has been found out that the general structure of the interaction network may play a crucial role in the emergence of synchronization phenomena in various fields such as physics, technology, and the life sciences.

Complex networks have already become an ideal research area for control engineers, mathematicians, computer scientists, and biologists to manage, analyze, and interpret functional information from real-world networks. Sophisticated computer system theories and computing algorithms have been exploited or emerged in the general area of computer mathematics, such as analysis of algorithms, artificial intelligence, automata, computational complexity, computer security, concurrency and parallelism, data structures, knowledge discovery, DNA and quantum computing, randomization, semantics, symbol manipulation, numerical analysis and mathematical software. This special issue aims to bring together the latest approaches to understanding complex networks from a dynamic system perspective. Topics include, but are not limited to the following aspects of dynamics analysis for complex networks: (a) synchronization and control, (b) topology structure and dynamics, (c) stability analysis, (d) robustness and fragility, and (e) Applications in real-world complex networks.

We have solicited submissions to this special issue from electrical engineers, control engineers, mathematicians, and computer scientists. After a rigorous peer review process, 17 papers have been selected that provide overviews, solutions, or early promises to manage, analyze, and interpret dynamical behaviors of complex systems. These papers have covered both the theoretical and practical aspects of complex systems in the broad areas of dynamical systems, mathematics, statistics, operational research, and engineering.

This special issue starts with a survey paper on the recent advances of multiobjective control and filtering problems for nonlinear stochastic systems with variance constraints. Specifically, in the paper entitled "Variance-constrained multiobjective control and filtering for nonlinear stochastic systems: a survey" by L. Ma et al., the focus is to provide a timely review on the recent advances of the multiobjective control and filtering issues for nonlinear stochastic systems with variance constraints. Firstly, the concepts of nonlinear stochastic systems are recalled along with the introduction of some recent advances. Then, the covariance control theory, which serves as a practical method for multiobjective control design as well as a foundation for linear system theory, is reviewed comprehensively. The multiple design requirements frequently applied in engineering practice for the use of evaluating 
system performances are introduced, including robustness, reliability and dissipativity. Several design techniques suitable for the multiobjective variance-constrained control and filtering problems for nonlinear stochastic systems are discussed. The design objects (nonlinear stochastic system), design requirements (multiple performance specifications including variance constraints), several design techniques and a special case of the addressed problem, mixed $\mathrm{H}_{2} / \mathrm{H}_{\infty}$ design problem, have been discussed in great detail with some recent advances. Subsequently, some latest results on the variance-constrained multiobjective control and filtering problems for nonlinear stochastic systems are summarized. Finally, concluding remarks are drawn and several possible future research directions are pointed out.

Complex networks have been extensively used in theoretical analysis of dynamical complex systems, such as the Internet, World Wide Web, communication networks, and social networks. Accordingly, the synchronization of dynamics complex networks has attracted a great deal of attention. In the paper entitled "Achieving synchronization in arrays of coupled differential systems with time-varying couplings" by $\mathrm{X}$. Yi et al., the synchronization problem is studied for complex dynamical networks described by linearly coupled ordinary differential equation systems. The time-varying coupling is used to represent the interaction between individuals. A general sufficient condition is derived such that the directed time-varying graph reaches consensus. Finally, a numerical simulation is provided to show the effectiveness of the theoretical results. The synchronization and reconstruction problem is investigated in "Chaos synchronization based on unknown input proportional multipleintegral fuzzy observer" by T. Youssef et al. for chaotic systems. An unknown input proportional multiple-integral observer is designed for synchronization of chaotic systems with immeasurable decision variables and unknown input. By using the Lyapunov stability theory and the linear matrix inequality (LMI) technique, sufficient conditions are given to ensure the synchronization. In the work entitled "Synchronization of switched complex bipartite neural networks with infinite distributed delays and derivative coupling" by Q. Bian et al., the synchronization problem is investigated for two coupled switched complex bipartite neural networks (SCBNNs) with distributed delays and derivative coupling. By constructing effective controllers, some synchronization criteria are proposed to ensure the synchronization of these two SCBNNs. The distributed consensus problem is studied in "Distributed impulsive consensus of the multiagent system without velocity measurement" by Z. Liu et al. for continuoustime multiagent system under intermittent communication. An impulsive consensus algorithm is developed, where the local algorithm of each agent is only based on the position information. Also, some necessary and sufficient conditions for consensus are given. Finally, a numerical example is given to illustrate the effectiveness of the theoretical analysis. Subsequently, in the paper entitled "Distributed consensus for discrete-time directed networks of multiagents with time-delays and random communication links" by Y. Liu et al., the leaderfollowing consensus problem is addressed for discrete-time directed multiagent systems with time-delay and random communication links. By constructing new Lyapunov functionals and employing some analytical techniques, sufficient conditions for the leader-following consensus in meansquare sense are established for multiagent system.

Over the past decades, the design of the controllers has long been the mainstream of research topics and much effort has been made for dynamical complex networks. In the paper entitled "Sliding intermittent control for BAM neural networks with delays" by J. Hu et al., the exponential stability problem is firstly studied for a class of delayed bidirectional associative memory (BAM) neural networks with delays. By taking the advantages of the periodically intermittent control idea and the impulsive control scheme, a sliding intermittent controller is designed for the addressed BAM system with time-delays. It is shown that such a sliding intermittent control method can comprise several kinds of control schemes as special cases. Also, some sufficient conditions are proposed such that the closed-loop delayed BAM neural networks are globally exponentially stable. The design of nonfragile gain-scheduled controller is discussed in "Nonfragile gain-scheduled control for discrete-time stochastic systems with randomly occurring sensor saturations" by $\mathrm{W}$. $\mathrm{Li}$ et al. for a class of discrete stochastic systems with randomly occurring sensor saturations (ROSSs). The sensor saturations occur in a random way. By constructing the probabilitydependent Lyapunov functional, a nonfragile gain-scheduled controller with the gain including both constant and timevarying parameters is designed such that the closed-loop system is exponentially stable in the mean-square sense. In the work entitled "Optimal guaranteed cost control of a class of discrete-time nonlinear systems with Markovian switching and mode-dependent mixed time-delays" by Y. Liu, the guaranteed cost control problem is addressed for a class of nonlinear discrete-time systems with Markovian jumping parameters and mixed time-delays. The mixed time-delays include both the mode-dependent discrete delay and the infinite distributed delay with mode-dependent lower bound. By constructing novel Lyapunov-Krasovskii functionals, some sufficient conditions for the existence of guaranteed cost controllers are derived. Also, a convex optimization approach is developed to minimize cost function so as to obtain the optimal guaranteed cost controller. By using the dwell time approach, in the paper entitled "Stabilization and controller design of $2 \mathrm{D}$ discrete switched systems with state delays under asynchronous switching" by S. Huang et al., the stability analysis and controller design are conducted for 2D discrete switched delayed systems represented by Roesser's model. Accordingly, sufficient conditions are given such that the resulting closed-loop systems are exponentially stable.

In the past years, the stability analysis of the dynamical complex networks has attracted much attention. In the work entitled "An analysis of stability of a class of neutral-type neural networks with discrete time delays" by Z. Orman and S. Arik, the problems of existence, uniqueness, and global asymptotic stability are discussed for a class of neutral-type neural network with discrete time-delays. By employing a Lyapunov functional and using the homeomorphism mapping theorem, some new delay-independent sufficient conditions are derived to guarantee the existence, uniqueness, and global 
asymptotic stability of the equilibrium point. It is shown that the advantage of the proposed results is that the developed results can be expressed in terms of network parameters only. Finally, some examples are provided to compare the proposed results with the existing results and to illustrate the effectiveness of the main results. In the work entitled "Robust almost periodic dynamics for interval neural networks with mixed time-varying delays and discontinuous activation functions" by $\mathrm{H}$. Wu et al., the delay-dependent robust exponential stability problem is studied for almost periodic solution of interval neural networks with mixed time-varying delays and discontinuous activation functions. According to the nonsmooth Lyapunov stability theory and employing the LMI technique, a new delay-dependent criterion is given to guarantee the existence and globally exponential stability of almost periodic solution. Also, the proposed results are extended to prove the existence and robust stability of periodic solution for neural networks with mixed time-varying delays and discontinuous activations. Finally, a numerical example is provided to show the feasibility of the developed results. The problem of bounded-input bounded-output (BIBO) stability is investigated in "BIBO stability analysis for delay switched systems with nonlinear perturbation" by J. Wei et al. for a class of switched systems with mixed neutral delays and nonlinear perturbation. By constructing the Lyapunov-Krasovskii functional, new BIBO stabilization criteria are derived. Finally, a numerical simulation is given to demonstrate the usefulness of the proposed results.

As is well known, the estimation theory has important applications in a variety of areas. In the work entitled "Deconvolution filtering for nonlinear stochastic systems with randomly occurring sensor delays via probability-dependent method" by Y. Luo et al., the deconvolution filtering problem is studied for a class of discrete-time stochastic systems with randomly occurring sensor delays and external stochastic noises. By constructing the probability-dependent Lyapunov functional and employing convex optimization approach, sufficient condition is given to ensure the stability of the addressed stochastic systems. The proposed gain-scheduled filters include both constant parameters and time-varying gains which can be updated online according to the measurable missing probabilities in real time. It is shown that the desired filters can be easily obtained by solving a set of linear matrix inequalities (LMIs). Finally, a simulation example is provided to illustrate the feasibility and effectiveness of the proposed filtering scheme. Subsequently, in the paper entitled "Estimate of number of periodic solutions of secondorder asymptotically linear difference system" by $\mathrm{H}$. Bin and $\mathrm{Z}$. Huang, the number of periodic solutions is discussed for second-order linear difference system. By using the Morse theory and twist number, three cases are discussed. As the system is resonant at infinity, the perturbation method is used to study the compactness condition of functional. Some new results are derived concerning the lower bounds of the nonconstant periodic solutions for discrete system. In the paper entitled "Convergence rate of numerical solutions for nonlinear stochastic pantograph equations with Markovian switching and jumps" by Z. W. Lu et al., the study of convergence rate is addressed for nonlinear stochastic pantograph equations with Markovian switching and Poisson jump. Sufficient conditions of existence and uniqueness of the solutions are given for nonlinear stochastic pantograph equations with Markovian switching and jumps. It is shown that Euler-Maruyama scheme for nonlinear stochastic pantograph equations with Markovian switching and Brownian motion is of convergence with strong order $1 / 2$. The meansquare convergence is preferable to be used for nonlinear stochastic pantograph equations with Markovian switching and pure jumps. Accordingly, the order of mean-square convergence is close to $1 / 2$. Moreover, the impact of human dynamics on the information propagation in online social networks is discussed in "Information propagation in online social network based on human dynamics" by Q. Yan et al. Also, an extended susceptible-infected (SI) propagation model is proposed to incorporate bursty human activity patterns and limited attention. The proposed result can be used to optimize/control the information propagation in online social networks.

\section{Acknowledgments}

This special issue is a timely reflection of the research progress in the area of nonlinear analysis of dynamical complex networks. We would like to acknowledge all authors for their efforts in submitting high-quality papers. We are also very grateful to the reviewers for their thorough and on-time reviews of the papers.

Zidong Wang Bo Shen

Hongli Dong Jun $\mathrm{Hu}$ 


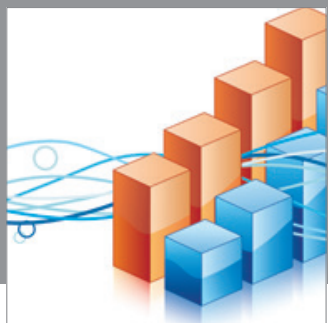

Advances in

Operations Research

mansans

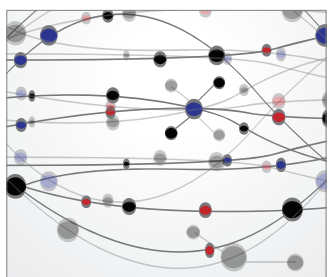

The Scientific World Journal
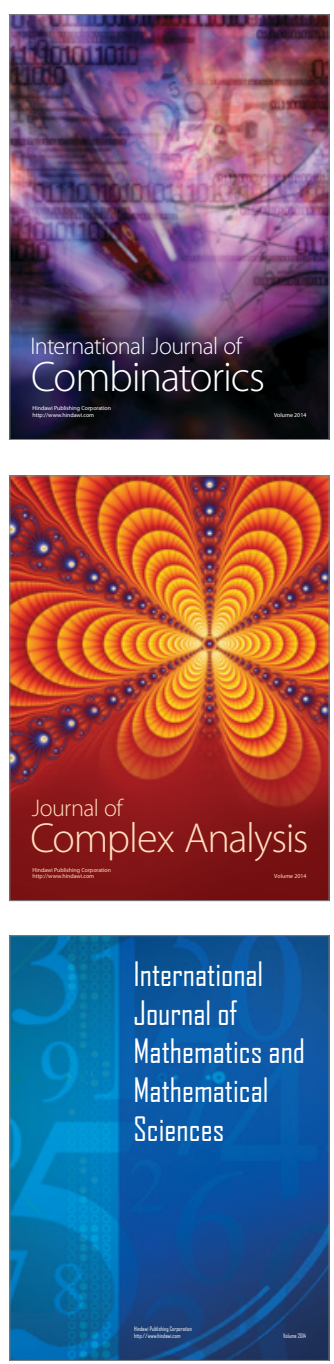
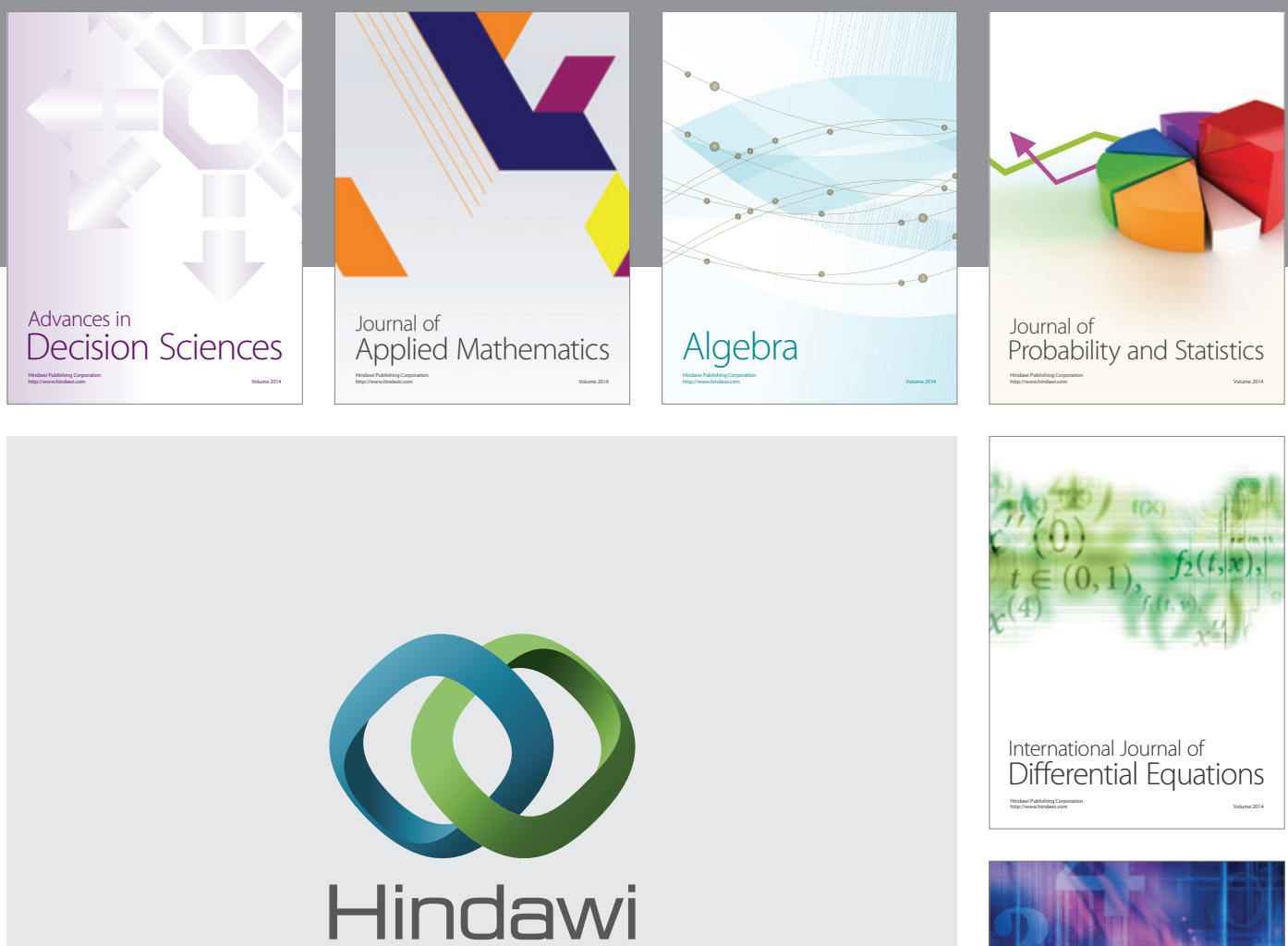

Submit your manuscripts at http://www.hindawi.com
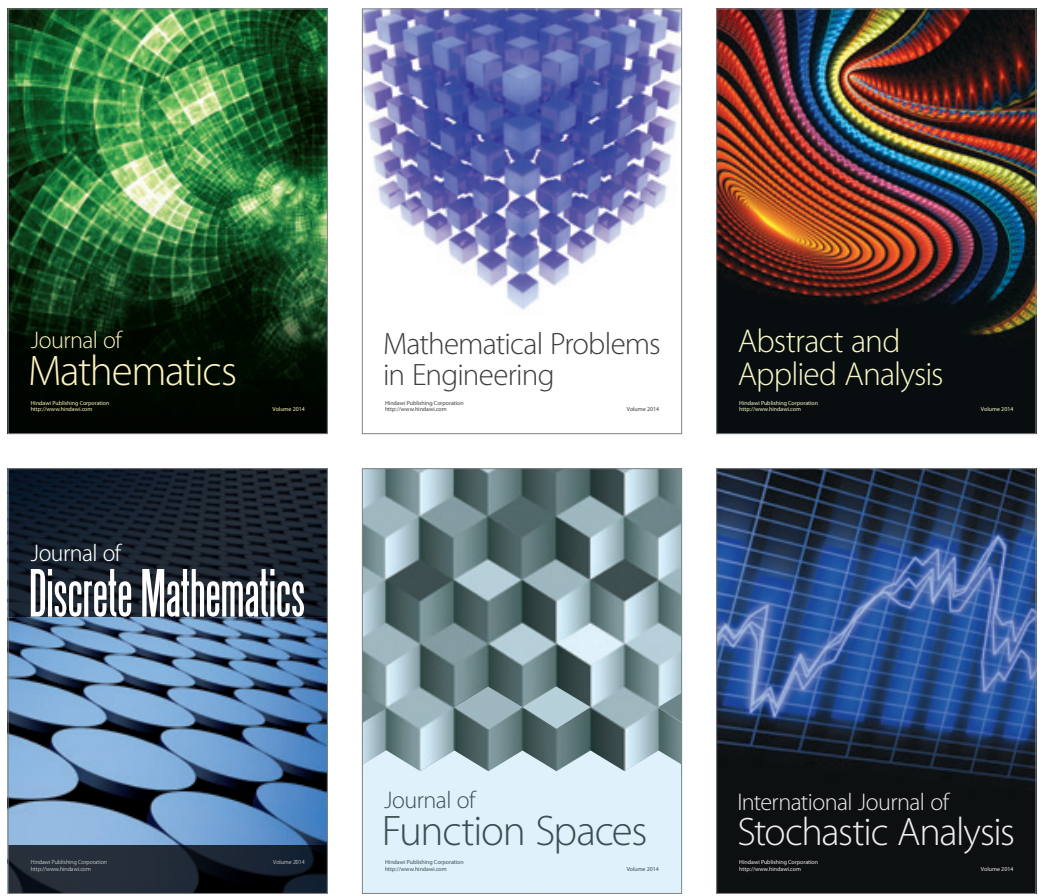

Journal of

Function Spaces

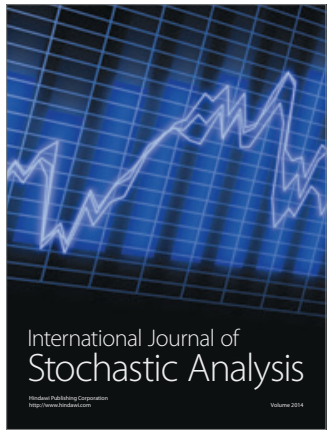

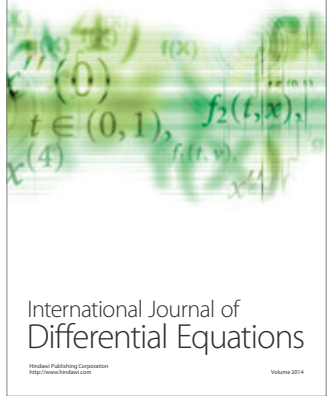
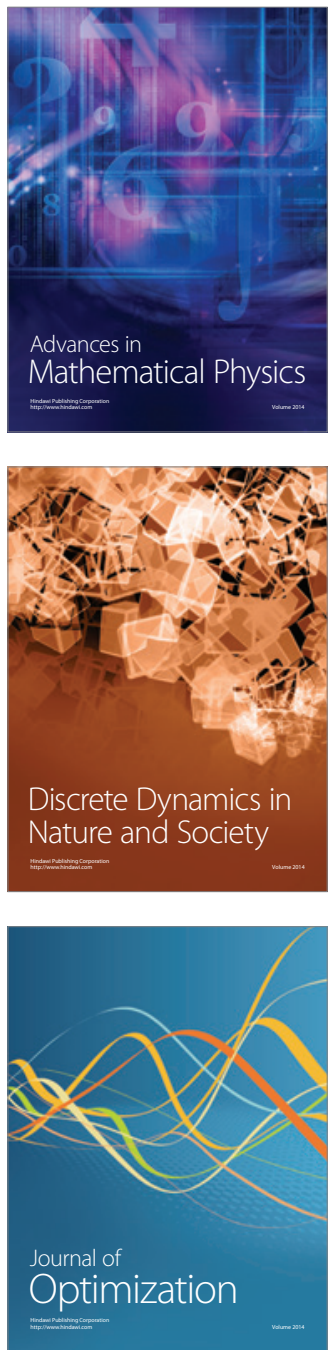